\title{
Flora Tristan oder die Wallfahrten einer Ausgestoßenen
}

Flora Tristan ist unter den in dieser Vorlesung gewählten reisenden Frauen sicherlich die international Bekannteste, was nicht nur an der Tatsache liegt, dass ihr der peruanische Literaturnobelpreisträger Mario Vargas Llosa mit El paraíso en la otra esquina einen halben Roman geschenkt hat, den sie sich mit ihrem noch berühmteren Enkel, dem französischen Maler Paul Gauguin, in Ehren teilt. Die Gründe für ihre eigene Berühmtheit sind darin zu sehen, dass Flora Tristan weit über die Grenzen Frankreichs hinaus als eine Vorkämpferin ebenso für die Frauenrechte wie für die Arbeiterrechte wahrgenommen wurde und ebenso innerhalb einer frühsozialistischen Umgebung wie einem feministischen Kontext sehr gut beleumundet ist. Doch der erfahrene Romancier Vargas Llosa hat schon im Titel zurecht das Paradies ins Spiel gebracht, war doch auch Flora Tristan (wie ihr Enkel) lange auf einer quasi-mystischen Suche nach einem Paradies für sich selbst wie für jene Gruppen, für die sie sich fast pausenlos in ihrem eher kurzen Erwachsenenleben einsetzte. Das Paradies, ihr Paradies, aber sollte sie selbst nie erreichen. Ihr Leben war eher das einer Ausgestoßenen, einer Paria.

Die 1803 in Paris geborene und bereits 1844 in Bordeaux verstorbene Flora Tristan war zweifellos eine der profiliertesten Frauen ihrer Zeit. Sie hat Geschichte gemacht und ihre Epoche aus heutiger Sicht mitgeprägt. Ihr Vater, ein wohlhabender peruanischer Adeliger, verstarb früh und ließ seine französische Frau und deren Tochter Flora mittellos zurück. Die behütete Kindheit war für Flora also rasch zu Ende und machte einem frühen Geldverdienenmüssen Platz.

Es war ein plötzlicher Sprung in ein Erwachsenenleben. Die junge Flora verdingte sich als Arbeiterin und ging mit achtzehn Jahren eine Vernunftehe mit ihrem damaligen Arbeitgeber, dem Lithographen André Chazal, ein. Dies war zweifellos die schlimmste Entscheidung ihres Lebens. Nach vier Jahren der Demütigung und ständiger Misshandlungen verließ Flora, die drei Kinder zur Welt gebracht hatte, von denen nur eine Tochter überlebte, ihren Mann, was im damaligen französischen Recht einem Ehebruch gleichkam. Die folgenden fünf Jahre war sie auf der Flucht ebenso vor ihrem Ehemann wie einer patriarchalischen französischen Justiz, die ihrem Noch-Gatten Recht gab. Sie lebte fortan in ständiger Angst; doch versuchte sie zugleich, ihre Tochter wie ihre Mutter zu ernähren.

Dies sind die Lehrjahre einer Frau, in der ein wachsendes politisches Bewusstsein sowohl für die Lage der Arbeiter wie auch der Frauen in der französischen Gesellschaft aufkeimte und schon bald einer präzisen politischen Diagnostik 


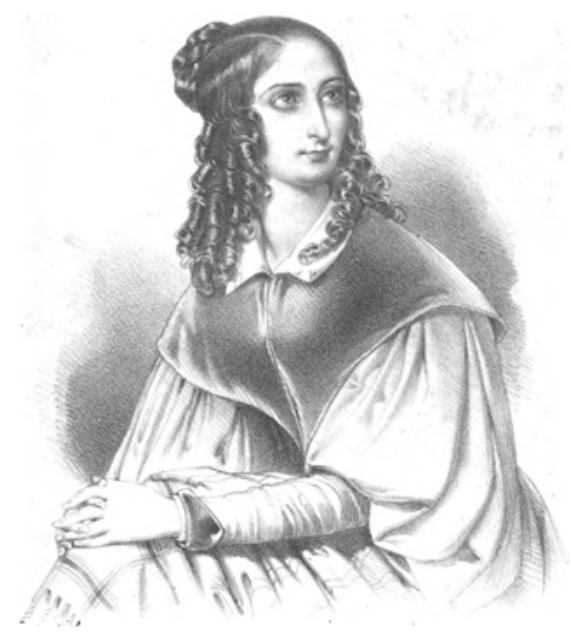

Abb. 81: Flora Tristan (Paris, 1803 - Bordeaux, 1844).

Raum gab. Letztere gewann an ständig zunehmender Schärfe gepaart mit einer aufreibenden Suche nach dem eigenen Ich als selbstbestimmte Frau.

Bald schon sollten den Lehrjahren Floras Wanderjahre folgen. Sie setzte große Hoffnungen auf den peruanischen Teil ihrer Familie und schiffte sich an ihrem dreißigsten Geburtstag in Bordeaux nach Peru ein. Sie war die einzige Frau an Bord. Flora gelangte in der Tat zu ihrer wohlhabenden peruanischen Familie und lernte die reichen Besitzungen wie auch den Einfluss ihrer männlichen Familienmitglieder kennen, interessierte sich zugleich aber auch für das Schicksal der Ärmsten im Lande, der Sklaven auf den Plantagen und der Frauen und Nonnen in einer vom peruanischen Patriarchat geprägten Gesellschaft. Aus ihrem Reisebericht geht deutlich hervor: Sie war eine unverbesserliche Rebellin.

In ihrem 1837 in zwei Bänden erschienenen literarischen Reisebericht, den Pérégrinations d'une paria, beschrieb sie in einer Mischung aus Reiseliteratur und Desillusionsroman, Sittenbeschreibung und Gesellschaftsanalyse, poetischen Landschaftsbeschreibungen und philosophischen Reflexionen ihre Erfahrungen während ihres mehrmonatigen Aufenthalts in Arequipa. ${ }^{1}$ Doch an

1 Vgl. u. a. Wolfzettel, Friedrich: 'Ce désir de vagabondage cosmopolite'. Wege und Entwicklungen des französischen Reiseberichts im 19. Jahrhundert. Tübingen: Max Niemeyer Verlag 1986, u. a. S. 139-146; Sánchez, Luis Alberto: Flora Tristán. Una mujer sola contra el mundo. Caracas: Biblioteca Ayacucho 1992; sowie sowie Hervé, Florence (Hg.): Flora Tristan oder: Der Traum vom feministischen Sozialismus. Berlin: Dietz 2013. 
eine Rückgewinnung ihres Erbes war für die Rebellin nicht zu denken: Sie war ihrer gesamten reichen Familie wesensfremd und musste sich bereits im Juli 1834 wieder nach Frankreich einschiffen - unverrichteter Dinge und so arm wie eh und je. Das peruanische Abenteuer war gescheitert.

Nach Paris und in die elenden Zwänge ihres Daseins zurückgekehrt, setzte sie alles daran, den Bericht über ihre Reise niederzuschreiben und im Jahre 1837 zu veröffentlichen. Dabei eröffnet ihr Reisebericht explizit die Sichtweise einer Frau, reflektiert deren Situation schonungslos und macht immer wieder auf die gesamtgesellschaftlichen Zustände einer bis vor kurzem noch kolonialen Gesellschaft aufmerksam, deren Machtverhältnisse und Sozialstruktur sich neu definieren. Gerade der Rolle der Frauen schenkt sie in diesem gesellschaftlichen Prozess größte Aufmerksamkeit.

Dabei fasziniert ebenso der kompositorische Charakter ihrer reiseliterarischen Darstellungen, die ganz im romantischen Duktus mit einer höchst subjektiven und zugleich poetischen Selbstreflektion beschäftigt sind, wie die klare, bisweilen schneidende Intelligenz einer zutiefst herausgeforderten Frau, welche die Ränkespiele ihrer Verwandtschaft rasch zu durchschauen lernt, ohne sich doch gegen diese zur Wehr setzen zu können. Sie hatte ihr Spiel in Peru von Beginn an verloren und gehörte keineswegs, wie gehofft, zu einer Familie, die sie nicht wollte und welche die Legitimität der Ehe ihres Vaters mit ihrer Mutter nicht anerkannte. Der einflussreiche Onkel ließ sich von ihr nicht erweichen: Das erhoffte Erbe blieb ihr unzugänglich, ihr Bittgang und ihre 'Pilgerschaft' bleiben ergebnislos. Sie selbst wird nun endgültig zu einer Ausgestoßenen, zu einer Paria.

Ich versage es mir an dieser Stelle, auf die Parallelen des Paria-Begriffes bei Flora Tristan und bei Hannah Arendt bezüglich Rahel Varnhagen aufmerksam zu machen. Auch wenn Flora Tristan, anders als Rahel Levin Varnhagen, nichts mit dem Judentum zu tun hatte, ${ }^{2}$ sind die Homologien zwischen den Frauenfiguren offenkundig.

Der gesamte Reisebericht zeichnet auf diese durchaus dramatische Weise einen Erkenntnisprozess nach, der mit vielen autobiographischen Elementen angereichert wird und einen Verlaufscharakter besitzt, der von der Ich-Erzählerin immer wieder kritisch reflektiert wird. Dabei schwanken die Beobachtungen der jungen Französin zwischen dem Ethnozentrismus ihrer eigenen Pariser Herkunft, die alles mit der Pariser Elle zu beurteilen versucht, und einer kritisch reflektierten Analyse der ökonomischen, politischen, aber vor allem sozialen und kulturellen Strukturen, mit denen sie auf ihrer Reise in Berührung kam. Es ist ein

2 Vgl. Ette, Ottmar: Mobile Preußen. Ansichten jenseits des Nationalen, Kap. 6. 
Reisebericht, der auf seiner autobiographischen Ebene von einem wachsenden Bewusstsein und vor allem Selbstbewusstsein zeugt.

Flora Tristans Reiseliteratur steht im Zeichen eines persönlichen wie strukturellen Scheiterns als Frau, wobei sie selbst sich als Gescheiterte dadurch zugleich auch als gescheiter erweist. Die subjektiven, zum Teil intimen autobiographischen Elemente verbinden sich mit Momenten der Memoirenliteratur, insofern sie etwa wichtige Protagonisten des in Peru entbrannten Bürgerkrieges von 1834 persönlich kennenlernt und über die persönlichen Implikationen dieser Figuren berichtet. So gerät der Reisebericht zu einem historischen Fresko von großer individueller wie kollektiver Relevanz, schildert die junge Französin doch nicht nur ihr eigenes Schicksal, sondern entwirft ein historisches Sittengemälde Perus und zugleich jener subalternen Situation, innerhalb derer sie sich als Frau wie eine Paria fühlen musste - auf beiden Seiten des Atlantik.

Fassen wir all jene biographischen Aspekte, die sich an die Abfassung des Reiseberichtes anschließen, in der gebotenen Kürze zusammen, um uns danach der Analyse ihrer Reiseliteratur zu widmen. Als Chazal von Flora Tristans Rückkehr nach Frankreich erfuhr, bedrohte er sie mehrfach und entführte schließlich ihre gemeinsame Tochter Aline, die zukünftige Mutter Paul Gauguins. Die französische Justiz sprach dem Vater freilich ungerührt das Sorgerecht zu. Als Flora durch herausgeschmuggelte Briefe schließlich von ihrer Tochter Aline erfuhr, dass sie vom Vater wiederholt missbraucht worden war, ging Flora gegen ihren Mann gerichtlich vor. Erst jetzt erlangte sie nach langem Kampf das Sorgerecht. Daraufhin lauerte ihr Chazal am 4. September 1838 auf und versuchte, seine frühere Frau auf offener Straße durch gezielte Pistolenschüsse zu töten. Flora überlebte nur knapp, eine Kugel blieb so nahe am Herzen stecken, dass sie nicht mehr entfernt werden konnte. Im nachfolgenden Prozess wurde Chazal zu Deportation und zwanzig Jahren Zwangsarbeit verurteilt, was es seiner Frau endlich erlaubte, sich legal von ihrem Mann scheiden zu lassen und fortan im Rahmen der gesetzlichen Möglichkeiten ein freies Leben als geschiedene Frau zu führen.

Flora Tristan widmete sich fortan ihrem politischen Kampf als Frühsozialistin und Feministin. Sie veröffentlichte Artikel und umfangreichere Schriften über das Los der Arbeiter und insbesondere die Situation der Frauen, die sie in Werkstätten, Fabriken und Bordellen besuchte. Ihre Berichte - nicht nur aus Frankreich, sondern auch aus England - rüttelten auf: Ihr englischer Reisebericht Promenades dans Londres, ou l'aristocratie et les prolétaires anglais erschien bereits 1840 und ist bis heute ein bewegendes Zeugnis der gesellschaftlichen Zustände im Mutterland der Industriellen Revolution.

Die beiden Jahre vor ihrem Tod verbrachte sie in Kutschen und auf Reisen kreuz und quer durch Frankreich, wo sie unentwegt Vorträge hielt, zu 
Zusammenschlüssen von Arbeitern aufforderte und das kritische Bewusstsein arbeitender Frauen förderte. Sie wurde von ihren Anhängern in kleinen Zirkeln gefeiert, wurde von der konservativen Presse verhöhnt und von der Polizei als gefährliches, aufwieglerisches Subjekt verfolgt. Vor Erschöpfung brach sie schließlich in Bordeaux zusammen und starb an Entkräftung und Typhus im Alter von einundvierzig Jahren.

Flora Tristans gesamtes Leben bietet das Beispiel einer mutigen, rastlosen und von ihrer Sache geradezu besessenen Frau, die ihre Zuhörer durch ihre Reden begeistern konnte, ihr Lesepublikum durch eine Mischung aus präziser Beschreibung und persönlicher Reflexion bestach und die buchstäblich für ihre Ideen lebte und sich verzehrte. In ihrem Reisebericht gibt es sehr wohl messianische, aber auch damit einhergehende agonale Züge, die sich auch in ihrem Leben mischten. Stets steht der Gewinn von Erkenntnis im Mittelpunkt all ihres Handelns, oder anders: Erkenntnis war der Antrieb für ihr Handeln. Dabei fand Flora Tristan, wie wir noch sehen werden, am Ende ihrer Pérégrinations Formulierungen für die Kraft und den Mut, für ihre Ziele zu kämpfen und dies, wenn notwendig, auch ganz alleine zu tun.

Flora rechnete sich selbst $\mathrm{zu}$ den besonders willensstarken Naturen - vielleicht wurde ihr gerade dies angesichts ihrer Überanstrengung am Ausgang eines allzu kurzen Lebens zum Verhängnis. Denn sie kämpfte nicht nur für ihre eigene Selbstbefreiung, sondern zugleich für die der Frauen in Frankreich und Europa sowie für die unterdrückte Arbeiterklasse ganz allgemein. Lange nach ihrem Tod erschien 1973 ein weiterer Bericht aus ihrer Feder, der von ihren letzten Fahrten im Auftrag der Union Ouvrière zeugt und unter dem Titel Le tour de France. Etat actuel de la classe ouvrière sous l'aspect moral, intellectuel, matériel erschienen ist. Doch ihr eigentlicher, bis heute berühmter Reisebericht bringt uns ihre Reise nach Peru nahe; wir wollen uns folglich mit dieser Reise und diesem Bericht beschäftigen und einmal mehr sehen, wie das Verhältnis von Reisen und Schreiben bei der französischen Autorin angelegt ist.

Gewiss kann man Flora Tristan in ihren Pérégrinations d'une paria einen deutlichen Eurozentrismus ankreiden; aber zugleich sind doch ihre Empathie mit den Unterdrückten und ihre Sympathie für die Frauen in Peru beeindruckend. Sie konnte ihr Bewusstsein als Europäerin sehr wohl als eines der eigenen zivilisatorischen Überlegenheit ausleben, hatte aber doch stets ein offenes Herz für all jene, die als Plantagensklaven oder als einfache Arbeiter schuften mussten. Ihr soziales Engagement geht mit ihrer romantisch eingefärbten Naturvorstellung - gerade auch in den Landschaftsszenen ihres Reiseberichts Hand in Hand. Aber sehen wir uns dies nun näher an.

Wir hatten uns ja bereits im theoretischen Teil unserer Vorlesung wiederholt mit den Pérégrinations d'une paria beschäftigt und erkannt, wie präzise konstruiert 
dieser reiseliterarische Text ist. So kann ich es mir an dieser Stelle erlauben, bestimmte Passagen herauszupicken, die für jene Fragestellungen von Bedeutung sind, auf die wir uns im Verlauf unserer Überlegungen noch etwas weniger eingelassen hatten. Dazu zählt an erster Stelle die neunte Dimension des Reiseberichts, die sich mit der genderspezifischen Darstellung und ihrer jeweiligen Perspektivik beschäftigt. Dabei sollen Aspekte in den Blick rücken, die wir bei Ida Pfeiffer und Fredrika Bremer noch weniger behandelt haben.

Flora Tristan geht in ihren Pérégrinations mehrfach auf die spezifische Rolle und die Schicksale jener Frauen ein, denen sie im Verlauf ihrer Reise begegnete. Da wäre etwa jene französische Gastwirtin zu Lima zu nennen, die Flora kennenlernte, weil sie nach Monaten keine Lust mehr verspürte, bei ihren Verwandten abzusteigen; ein Aufenthalt in einem Hotel verschaffte ihr zugleich eine größere und vor allem unbeaufsichtigte Bewegungsfreiheit, welche sie auch zu nutzen verstand. Diese Französin hatte sich äußerst aktiv in der Neuen Welt niedergelassen und ihr eigenes Hotel gegründet. Es gelang ihr so, jene Zeit zu verarbeiten, in der sie zunächst an der Pariser Oper bekannt geworden, später aber vom französischen Publikum fallengelassen worden war. Flora interessierte sich für ihre Geschichte. Denn die Französin hatte sich in der Neuen Welt ein neues Leben aufgebaut, das ihr auch neue Freiheiten als Frau schenkte.

Besonders aufschlussreich für uns aber sind jene Passagen, in denen sich Flora Tristan mit den berühmten Frauen von Lima beschäftigt, die für ihre freizügige und von Männern nicht zu kontrollierende Lebensweise bekannt waren. Bei allen europäischen Reisenden des 18. und 19. Jahrhunderts waren diese Frauen berühmt, weil sie nicht allein als ausnehmend schön, ja als Inbegriff weiblicher Schönheit galten, sondern weil sie nach verbreiteter Ansicht die kleinsten Frauenfüßchen besäßen, die den Europäern jemals zu Augen gekommen waren. Dies wurde zu einem wahren Topos, der in der entsprechenden Reiseliteratur zur Hauptstadt Perus immer wieder auftauchte und nicht auszurotten war. Drittens aber waren sie dafür bekannt und berüchtigt, dass sie sich auf der Straße zu verhüllen pflegten, so dass sie nicht erkannt werden konnten, nicht einmal von ihren eigenen Ehemännern. Sie konnten daher ein relativ freies, unbeaufsichtigtes und - wie man sagte - sittenloses Leben führen und ihren Neigungen stets nachgeben. Allein Alexander von Humboldt scheint unter den europäischen Reisenden von diesen Frauen wenig begeistert gewesen zu sein. Sehen wir uns jedoch einmal Flora Tristans Reaktion auf diesen lebendigen peruanischen Topos näher an.

Nach einer ausführlichen Beschreibung der weiblichen Kleidungsstücke, die viel zur Berühmtheit der Frauen von Lima in der europäischen Literatur beigetragen hatten, nämlich der Beschreibung von saya und manto, stellt Flora Tristan allgemeine Überlegungen zur weiblichen Mode an. Diese charakteristischen Kleidungsstücke verhüllten und zeigten zugleich, eine Funktionsweise weiblicher 
Mode, indem sie den gesamten weiblichen Körper, einschließlich des Gesichts und der Haare, mit Ausnahme eines der beiden Augen vollständig verhüllten; zugleich zeigten sie durch eine enorm zugeschnürte weibliche Taille und das Freilassen der Füßchen die körperlichen Reize der unbekannten Frau deutlich an und brachten ihre Körperformen zur Geltung.

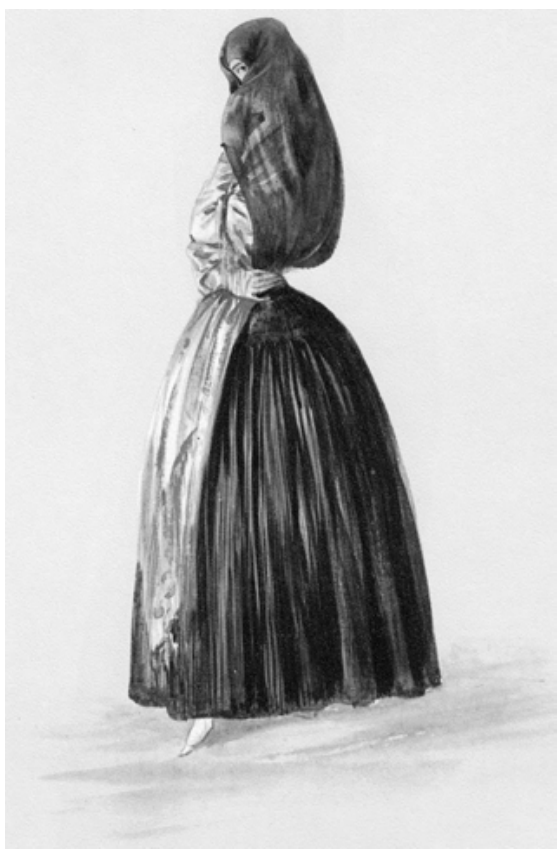

Abb. 82: „Tapada“, Aquarell von Johann Moritz Rugendas, 1843.

Man sieht bei dieser Gelegenheit, wie aufmerksam die Französin gegenüber den in Wert gesetzten Reizen, mehr aber noch der spezifischen Ausdrucksmittel der Mode der Bewohnerinnen von Lima war. Sie glauben mir das alles nicht? Dann möchte ich Ihnen zumindest einen kleinen Ausschnitt aus dieser Textpassage bieten.

Une des recherches de leur luxe est encore d'avoir un très beau mouchoir de batiste brodé garni de dentelle. Oh! qu'elles ont de grâce, qu'elles sont enivrantes ces belles Liméniennes avec leur saya d'un beau noir brillant au soleil, et dessinant des formes vraies chez les unes, fausses chez beaucoup d'autres, mais qui imitent si bien la nature, qu'il est impossible, en les voyant, d'avoir l'idée d'une supercherie! ... Qu'ils sont gracieux leurs mouvements d'épaules, lorsqu'elles attirent le manto pour se cacher entièrement la figure, que par instants elles laissent voir à la dérobée! Comme leur taille est fine et souple, 
et comme le balancement de leur démarche est onduleux! Que leurs petits pieds sont jolis, et quel dommage qu'ils soient un peu trop gros!

Une Liménienne en saya, ou vêtue d'une jolie robe venant de Paris, ce n'est plus la même femme; on cherche vainement, sous le costume parisien la femme séduisante qu'on a rencontrée le matin dans l'église de Sainte-Marie. ${ }^{3}$

Dieser Text gibt Aufschluss über die große Faszinationskraft, die von dieser andersartigen, exotisch und erotisch zugleich wirkenden Kleidung von Frauen ausgeht, die in einem fremden, ungewohnten Kontext wahrgenommen werden. Flora Tristan unterstreicht die Wirkung weiblicher Achtsamkeit durch Zitate von Männern, die beim Anblick derartiger Schönheiten nachgerade zu Abenteurern würden. Selbstverständlich ist es evident, dass derartige Darstellungen mit einer gehörigen Dosis Orient und mehr noch Orientalismus gewürzt werden, ist doch auch der Orient in der abendländischen Imagination voller zauberhafter verschleierter Frauen, die der männliche Blick auf den weiblichen Körper so gerne entschleiern möchte. Flora Tristan befriedigt in dieser Passage durchaus einen gewissen Voyeurismus ihrer europäischen Zeitgenossen und auch ihrer Zeitgenossinnen, die in aller Regel mit dem Topos der Frauen von Lima bestens vertraut waren.

So geht es hier nicht zuletzt um das europäische Lesepublikum, das direkt angesprochen und gleichsam bei den Bildern der eigenen Imagination abgeholt werden soll. Flora Tristan bedient alle Stereotypen der europäischen Reiseliteratur, so wie uns der deutsche Maler Johann Moritz Rugendas, mit dem wir uns noch kurz beschäftigen werden, ebenfalls ein recht anschauliches Bild der Tapadas, der verhüllten Frauen von Lima, zu bieten versteht.

Die Französin versteht etwas von weiblicher Mode und Kleidung; sie beschreibt gekonnt die einzelnen Bestandteile derselben und gibt in ihrer literarischen Darstellung auch der Wirkung auf die Betrachter reichlichen Ausdruck. Doch nach der ausführlichen Darstellung der Tapadas wäre die Rebellin (oder nach eigenen Worten die Paria) Flora Tristan nicht Flora Tristan, hätte sie nicht am Ende eine kleine, aber zerstörerische, ja vernichtende Kritik eingebaut und den zwar kleinen, aber doch etwas zu dicken Fuß der Limeñas ins literarische Spiel gebracht. Sie wäre aber auch nicht sie selbst gewesen, hätte sie nicht ausführlich Bezug genommen auf den sozialen Status der Frauen und all jene Möglichkeiten, die ihnen ihre schwarze Kleidung gab, die gleichsam das Gegenteil eines Witwenkostüms darstellte.

$\mathrm{Zu}$ diesem Gesamtkomplex möchte ich Ihnen an dieser Stelle eine etwas längere Passage im Auszug vorstellen, um zugleich auch darauf hinzuweisen,

3 Tristan, Flora: Pérégrinations d’une paria, S. 333: 
dass die Bewohnerinnen von Lima nicht allein mit den Orientalinnen, sondern auch mit den Europäerinnen verglichen werden. Dieser Vergleich wirft ein bezeichnendes Licht auf die Rolle und den Status der Frauen in Europa. Hier wird die emanzipatorische Intentionalität Flora Tristans, ihres gesamten Schreibens wie vor allem auch ihres faszinierenden Reiseberichts unverkennbar deutlich. So schreibt die französische Autorin:

\begin{abstract}
Cependant les femmes de Lima gouvernent les hommes, parce qu'elles leur sont bien supérieures en intelligence et en force morale. La phase de civilisation dans laquelle se trouve ce peuple est encore bien éloignée de celle où nous sommes arrivés en Europe. Il n'existe au Pérou aucune institution pour l'éducation de l'un ou de l'autre sexe; l'intelligence ne s'y développe que par ses forces natives: ainsi la prééminence des femmes de Lima sur l'autre sexe, quelque inférieures, sous le rapport moral, que soient ces femmes aux Européennes, doit être attribuée à la supériorité d'intelligence que Dieu leur a départie. [. . . ]

D'après ce que je viens d'écrire sur le costume et les usages des Liméniennes, on concevra facilement qu'elles doivent avoir un tout autre ordre d'idées que celui des Européennes, qui, dès leur enfance, sont esclaves des lois, des mœurs, des coutumes, des préjugés, des modes, de tout enfin; tandis que, sous la saya la Liménienne est libre, jouit de son indépendance et se repose avec confiance sur cette force véritable que tout être sent en lui, lorsqu'il peut agir selon les besoins de son organisation. La femme de Lima, dans toutes les positions de la vie, est toujours elle; jamais elle ne subit aucune contrainte: jeune fille, elle échappe à la domination de ses parents par la liberté que lui donne son costume; quand elle se marie, elle ne prend pas le nom de son mari, garde le sien, et toujours reste maitresse chez elle [...].

J'ai dépeint les femmes de Lima telles qu'elles sont et non d'après le dire de certains voyageurs [...]; mais mon rôle de voyageuse consciencieuse me faisait un devoir de dire toute la vérité. ${ }^{4}$
\end{abstract}

In diesem Zitat findet sich eine Vielzahl von Feststellungen, Behauptungen und Überzeugungen, welche auf eine durchaus überraschende Art und Weise den Unterschied zwischen den Frauen von Lima und den Europäerinnen - wobei Flora vor allem an die Französinnen dachte - ausleuchten. Dabei grenzt sich Flora Tristan explizit von den Stellungnahmen früherer (und männlicher) Reisender ab, welche vor allem den erotischen Zug der Limanerinnen und ihre sexuelle Freizügigkeit, aber auch ihre große Schönheit betonten. Bei ihr hingegen liegt der Schwerpunkt der Darstellung ganz eindeutig auf der Intelligenz dieser Frauen, die weit höher sei als jene ihrer Herrscher und Gebieter. Sie sind nicht schöner, aber intelligenter.

Überdies findet sich in diesem Zitat ein Vergleich mit den Europäerinnen, der - auf den ersten Blick aufgrund des Eurozentrismus Flora Tristans überraschend - zu Ungunsten der europäischen Gesellschaften ausfällt. Zwar seien

4 Ebda., S. S. 335-340. 
diese Gesellschaften weiter auf dem Weg der Zivilisation vorangeschritten und die junge Französin glaubt nicht weniger als ihre Zeitgenossen an die Existenz eines einzigen Weges der Zivilisation und der Modernisierung, und von derlei Gläubigen wird noch heute die Mehrzahl unserer europäischen Länder besiedelt -, doch macht sie darauf aufmerksam, dass die Frauen eigentlich eine höhere Intelligenz ins Leben mitbringen.

Die Argumente Flora Tristans sind präzise und scharf: Es seien die gesellschaftlichen Institutionen, allen voran jene von Bildung und Erziehung, es seien weiterhin die Eltern, die Abhängigkeit von den Ehegatten, der Verzicht auf gleichen Zugang zum Gesetz, schließlich aber auch die Unterdrückung in den öffentlichen Verhaltensweisen und vieles mehr, was die untergeordnete Rolle der Frauen in den Ländern Europas verursacht habe. Die Unfreiheit der Europäerinnen sei daher vor der Hintergrundfolie der Frauen von Lima mit Händen zu greifen - eine Situation der europäischen Frau, die nicht nur beklagenswert, sondern in den Augen der kämpferischen Französin sehr wohl veränderbar sei.

Auch die Thematisierung der Frage der Familiennamen, die natürlich nicht nur bei den Bewohnerinnen von Lima dazu führt, dass der Familienname des Ehegatten nicht 'angenommen' wird, dient vor allem dazu, eine Gewohnheit und einen Anspruch der Männer gegenüber den Frauen in Europa zu untergraben, gibt es doch eigentlich keinen vernünftigen Grund, warum generell der Name des Mannes den der Frau auslöschen sollte. Dies alles kleidet Flora Tristan ein in die Behauptung, sich nicht an den bisherigen Aussagen anderer Reisender und anderer Reiseberichte ausgerichtet und orientiert zu haben. Sie behauptet demgegenüber, auch mit ihren vielen kritischen Bemerkungen, die sie überall in ihren Text einstreute, eine gewissenhafte Reisende zu sein, die ihren Leserinnen und Lesern die Menschen wie die Gegenstände genau so präsentiert, wie sie wirklich seien.

So findet sich auch bei Flora Tristan der umfassende Wahrheitsanspruch des Reiseberichts in expliziter Form. Interessanterweise ist dies gerade dort der Fall, wo es um die Geschlechterbeziehungen in einem weit von Frankreich entfernten Land geht, deren Betrachtung und Erörterung gleichwohl viele Rückschlüsse auf die eigene Gesellschaft zulässt. So kann Flora Tristan durchaus als eine der ersten Reisenden bezeichnet werden, die gleichsam mit einem emanzipatorischen feministischen Projekt, in jedem Falle aber mit einem gegenüber den Geschlechterbeziehungen besonders geschärften Blick ihre Reise nach Amerika antrat und durchführte. Dies war insgesamt fraglos ein Novum innerhalb der langen Geschichte der Reiseliteratur. Denn die Frauen führten nun selbst nicht mehr nur ihre eigenen Reisen durch, sondern zogen auch weltweit Vergleiche, die ihre Situation in Europa kritisch beleuchteten. 
Flora Tristans Position ist hierbei nach eigenem Bekunden die der Paria, der Außerhalbbefindlichen, der Ausgestoßenen. Wie wir aus ihrer Biographie wissen, standen ihr in Frankreich die schlimmsten Auseinandersetzungen mit ihrem Mann wie auch mit der französischen Justiz ja noch bevor. Wenn wir diese Position mit der von Hannah Arendt in ihrem Buch über Rahel Varnhagen ins Spiel gebrachten - wenn auch nicht von ihr erfundenen - Unterscheidung zwischen Paria und Parvenü in Verbindung bringen, dann ist es eben diese Paria-Position der Außerhalbbefindlichkeit, welche es der jungen Französin erlaubt, ihre eigene französische Gesellschaft, aber auch andere Gesellschaften kritisch von außen zu bewerten und gleichsam von höherer, in jedem Falle aber anderer Warte aus darzustellen. Die Position der Paria war folglich für Flora trotz aller schmerzhaften Begleiterscheinungen eine Position der Unabhängigkeit und Freiheit.

Die Reise Flora Tristans begann an ihrem dreißigsten Geburtstag, und sie wird von Flora so geschildert, als sei sie aus der Mitte der Gesellschaft in Frankreich förmlich vertrieben worden. In der Tat hatte sie zum damaligen Zeitpunkt als Frau bereits alle Benachteiligungen, Beschränkungen, Demütigungen und selbst sexuellen Ausbeutungen erfahren, was ihr die Augen für ihre Zeit öffnete. Vor diesem ganz konkreten Hintergrund der Verfolgungen durch ihren Mann und durch die französische Justiz entsteht ihre Position der Paria, der Vertriebenen, der Exilierten, der Ausgestoßenen, die von Beginn an eingenommen wird und sich bis zum Schluss der Reise kontinuierlich durchzieht. Doch wo ist Hilfe für die junge Frau?

Ungezählte Male ruft sie in ihrem Reisebericht Gott an, wendet sich an ihren Schöpfer und hofft auf seine Antwort und auf sein Eingreifen - so auch bei der Durchquerung jener Wüste, die wir uns im theoretischen Teil unserer Vorlesung bereits näher angeschaut hatten. Auch am Ende ihrer Reise erscheint die Anrufung an ihren Gott ein letztes Mal. So gibt es vielleicht doch noch einen männlichen Blick, der transzendent alle irdischen Dinge im Reisebericht quert? Und so ist es auch eine innere Stimme, die ihr trotz aller Selbstmordphantasien von Beginn an sagt, dass sie nicht sterben werde, dass sie vielmehr leben werde, um ihr Schicksal zu vollenden.

Die Reise ist für die junge Frau ein einziger langer Weg der Erkenntnis. Gleich bei ihrer ersten Station, einem durch technische Probleme erzwungenen Aufenthalt auf den Kapverdischen Inseln und dort in La Praya, werden uns Vorstellungen präsentiert, die sehr anschaulich und detailliert zum einen das Auseinanderklaffen zwischen dem erzählten oder reisenden Ich und dem erzählenden oder wieder nach Frankreich zurückgekehrten Ich aufweisen. Zum anderen erfolgt eine deutliche Öffnung der nationalistischen Vorstellungen der Französin hin zu einer Art Weltbürgertum, einem weltläufigen Kosmopolitismus, wie er im 18. Jahrhundert 
wesentlich stärker vorhanden gewesen, aber dann durch die im napoleonischen und nachnapoleonischen Zeitalter aufkommenden Nationalismen weitgehend verdrängt worden war. Floras Erkenntnisprozess schließt die Notwendigkeit mit ein, weit über die Grenzen Frankreichs hinaus zu denken.

Lassen Sie mich diese Passage an das Ende unserer Beschäftigung mit der Reiseliteratur von Frauen im 19. Jahrhundert stellen, wird hier doch eine ihrer Wirkungen greifbar, die bisweilen erzielt werden kann: die Einsicht in ein Lebenswissen, das jenseits der eng gezogenen nationalen Grenzen auf eine globale Perspektive aufmerksam wird, auf Zusammenhänge und Solidaritäten, die das Zusammenleben, die Konvivenz der Menschheit auf dem gesamten Planeten betreffen. Dass sich hier Zusammenhänge mit der sozialistischen Idee einer weltweiten Verbindung der Arbeiterschaft ergeben, ist vor dem Hintergrund ihrer späteren politischen Entwicklung evident.

Doch hieran haben auch die Reise und der Reisebericht ihren Anteil. Denn auch dies ist die transatlantische Reiseliteratur: die Entstehung eines Bewusstseins für ein Zusammenleben der Menschen in Frieden und in gegenseitigem Respekt vor der Differenz der jeweils Anderen. Auch in diesem Sinne ist die Reise daher ein tiefer Erkenntnisprozess, der die Relation zwischen Reisen und Schreiben in einen philosophischen Erkenntniszusammenhang stellt und jene Veränderungen konstatiert, die vom Reisen selbst ausgelöst wurden und das Denken wie das Schreiben prägen.

En 1833, j'étais encore bien loin d'avoir les idées qui, depuis, se sont développées dans mon esprit. A cette époque, j'étais très exclusive: mon pays occupait plus de place dans ma pensée que tout le reste du monde; c'était avec les opinions et les usages de ma patrie que je jugeais des opinions et des usages des autres contrées. Le nom de la France et tout ce que s'y rattachait produisaient sur moi des effets presque magiques. Alors je considérais un Anglais, un Allemand, un Italien comme autant d'étrangers: je ne voyais pas que tous les hommes sont frères et que le monde est leur commune patrie. J'étais donc bien loin encore de reconnaître la solidarité des nations entre elles, d'où résulte que le corps humanitaire en entier ressent le bien et le mal de chacune d'elles. ${ }^{5}$

In Flora Tristan ist durch die Reise eine Veränderung ihrer eigenen Position nachhaltig in Bewegung gekommen. Die physische Bewegung auf der Erdoberfläche brachte bei ihr auch eine mentale Bewegung ihrer Positionalität hervor, in welcher zugleich die Mobilität vorherrscht. Die Außerhalbbefindlichkeit der Paria gegenüber ihrem eigenen Land verwandelt sich in eine Extraterritorialität auf einer globalen Ebene, die fortan in ihrem Denken eine wesentliche Rolle spielen wird. Dies geht mit einer deutlichen Kritik an ihrer früheren, nationalistischen Position einher, die sie nun über Bord zu werfen gewillt ist.

5 Ebda., S. 23. 
Über Bord gingen zugleich auch andere Illusionen. Etwa die, in ein gleichberechtigtes Leben in Frankreich zurückkehren zu können. Flora Tristans Reisebericht geht mit dem Ablegen von der peruanischen Küste zu Ende und nicht mit der Rückkehr in ihr Heimatland. Ihre Entschlossenheit lange vor der reiseliterarischen Rückkunft nach Frankreich wird in den Schlußworten ihres Reiseberichts deutlich: „on leva l'ancre; tout le monde se retira; et je restai seule, entièrement seule, entre deux immensités, l'eau et le ciel““. ${ }^{6}$ Die Einsamkeit des Ich am Ende des Reiseberichts ist keineswegs nur jene des romantischen Ich im Angesicht der Schöpfung; denn diese Einsamkeit wird die Einsamkeit einer Kämpferin sein, die für sich zurecht reklamieren darf, ihre Rolle als Paria in Hinblick auf die Gestaltung der Zukunft vollständig ausgefüllt zu haben.

6 Ebda., S. 377. 